\title{
LATENCY CONSIDERATIONS FOR ADAPTIVE MODULATION IN AN INTERFERENCE-FREE SLOW RAYLEIGH FADING CHANNEL
}

\author{
J. M. Torrance and L. Hanzo
}

\author{
Dept. of Electr. and Comp. Sc., Univ. of Southampton, SO17 1BJ, UK. \\ Tel: +44-1703-593 125, Fax: +44-1703-594 508 \\ Email: jmt94r@ecs.soton.ac.uk, lh@ecs.soton.ac.uk \\ http://www-mobile.ecs.soton.ac.uk
}

\begin{abstract}
Adaptive modulation exploits the time-variant channel capacity fluctuation of fading channels using a range of different modem modes according to Equation 1 and Table1. This is achieved at the cost of some latency penalty, which is quantified in this treatise and mitigated by frequency hopping.
\end{abstract}

\section{INTRODUCTION}

Steele and Webb [1] proposed adaptive modulation for mobile fading channels and recently there has been considerable interest in its performance [2]-[6]. The principle of operation is to employ the most appropriate Transmission Scheme (TS) in order to maintain a certain target bit error rate (BER) performance. The most appropriate TS is dependent upon the instantaneous Signal-to-noise Ratio (SNR), which varies as the channel experiences narrow-band fading. The TS that is used is selected as follows [3]:

TS $=\left\{\begin{array}{lll}\text { No Transmission } & \text { if } & l_{1}>s^{2} / N \\ \text { BPSK } & \text { if } \quad l_{1} \leq s^{2} / N<l_{2} \\ \text { QPSK } & \text { if } \quad l_{2} \leq s^{2} / N<l_{3} \\ \text { Square 16 Point QAM } & \text { if } \quad l_{3} \leq s^{2} / N<l_{4} \\ \text { Square 64 Point QAM } & \text { if } \quad s^{2} / N \geq l_{4},\end{array}\right.$

where $s$ is the instantaneous signal level, $N$ is the average noise power, and $l_{1}, l_{2}, l_{3}$ and $l_{4}$, are the BER-dependent optimised switching levels. Time Division Duplex (TDD) was proposed, in order to estimate the channel conditions on a slot by slot basis [2]. This requires up- and down-link slots in the TDD frame to be adjacent for every user, rather than employing the conventional configuration, where the downand up-link slots are in separate halves of the frame, which imposes various practical constraints. Furthermore, bringing the up- and down-link slots closer implies that now the channel correlation between the mobile reception and transmission is reduced. This problem can be mitigated by evaluating the received signal level of the TDD slot preceeding the mobile's up-link slot. Following this 'passive reception', up-link transmission takes place, which is the basis for the channel estimation for the down-link transmission, etc.

The BER upper-bound performance of such a scheme in a Rayleigh fading channel was given by Torrance [3]. Similar schemes have been investigated by Suzuki, Sampei

VTC'97, PHOENIX, USA, TECHNICAL AREA: MODULATION \& CODING

\begin{tabular}{|l|c|c|c|c|}
\hline BER & $l_{1}$ & $l_{2}$ & $l_{3}$ & $l_{4}$ \\
\hline $1 \cdot 10^{-2}$ & $3.31(\mathrm{~dB})$ & $6.48(\mathrm{~dB})$ & $11.61(\mathrm{~dB})$ & $17.64(\mathrm{~dB})$ \\
$1 \cdot 10^{-4}$ & $7.98(\mathrm{~dB})$ & $10.42(\mathrm{~dB})$ & $16.76(\mathrm{~dB})$ & $26.33(\mathrm{~dB})$ \\
\hline
\end{tabular}

Table 1: Optimised switching levels for speech and computer data systems through a Rayleigh channel, shown in dB

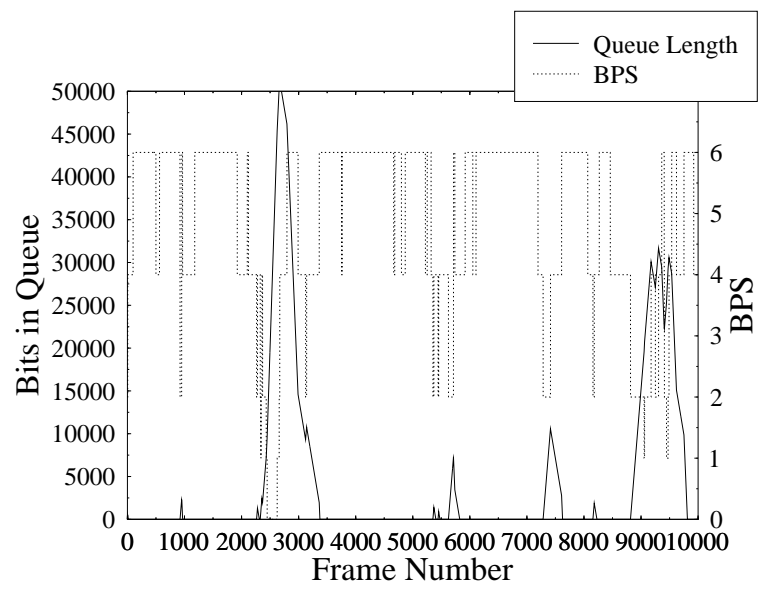

Figure 1: Instantaneous length of the buffer and the instantaneous throughput

and Morinaga [4] as well as Chau and Goldsmith [5]. It has been shown [6] that optimisation may be employed for example to ensure that BERs of $1 \times 10^{-2}$ and $1 \times 10^{-4}$ are maintained for average channel SNRs in the range of 0 to $50 \mathrm{~dB}$ over a Rayleigh fading channel, if the switching levels $l_{1}, l_{2}, l_{3}$ and $l_{4}$ are assigned as shown in Table 1 .

The lower and higher BER schemes are considered for speech and computer data transmission systems, respectively. However, the bitrate of both schemes fluctuates on a short term basis as a result of temporal variations in the modulation scheme that is employed. It is proposed to invoke a buffer at the input to the modulator, in order to smoothe the short term bitrate fluctuations. As an example, the instantaneous 'fullness' of the buffer and the instantaneous throughput are shown in Figure 1 as a function of the transmission frame index. It is clear that the buffer length has ramifications as to system latency.

Lower normalised Doppler frequencies result in high- 


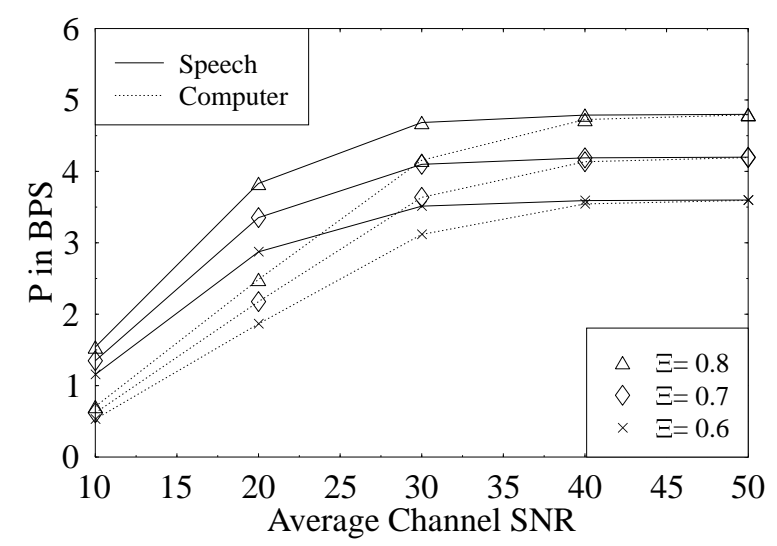

Figure 2: BPS performance in terms of $P$ for adaptive speech and computer data schemes, $\Xi$ values of $0.6,0.7$ and 0.8 and for a range of average channel SNRs.

er temporal channel correlation, which is exploited in order to estimate the channel quality and, therefore, employ the most appropriate modulation scheme. Ironically, lowDoppler channels result in prolonged fades that contribute to the adaptive modulation's latency.

Section 2 highlights the bit per symbol performance of the proposed adaptive modem, Section 3 outlines the the experimental conditions, while Section 4 presents a performance overview, before concluding in Section 5 .

\section{BITS PER SYMBOL PERFORMANCE}

The average throughput $B$ of an adaptive modulation scheme, which is expressed in Bits Per Symbol (BPS), was derived for a Rayleigh fading channel in Reference [3]:

$$
\begin{aligned}
B & =1 \cdot \int_{l_{1}}^{l_{2}} F(s, S) d s+2 \cdot \int_{l_{2}}^{l_{3}} F(s, S) d s \\
& +\quad 4 \cdot \int_{l_{3}}^{l_{4}} F(s, S) d s+6 \cdot \int_{l_{4}}^{\infty} F(s, S) d s,
\end{aligned}
$$

where $F(s, S)$ is the PDF of the Rayleigh channel and $S$ is the average power. In this contribution the actual average number of transmitted bits per symbol is $P$ is kept below the BER-specific maximum value $B$ in order to be able to invoke more robust modem modes for the sake of reducing the duration of 'No Transmission' intervals. We then define the relative BPS capacity as: $\Xi=P / B$. The average upper-bound BPS performance of an adaptive speech and computer data scheme for $\Xi$ values of $0.6,0.7$ and 0.8 is shown for a range of average channel SNRs in Figure 2.

\section{EXPERIMENTAL FRAME-WORK}

Adaptive modulation could be exploited within a Universal Mobile Telecommunications System (UMTS) air interface. One of the European UMTS air-interface proposals is due to the ACTS FRAMES project, proposing a multi-mode air interface [7]. This includes a TDD option with a $4.615 \mathrm{~ms}$ frame duration, up to 64 slots per frame for $2 \mathrm{GHz}$ transmission. Including adaptive modulation in this frame-work results in a temporal difference of $72 \mu$ s between channel

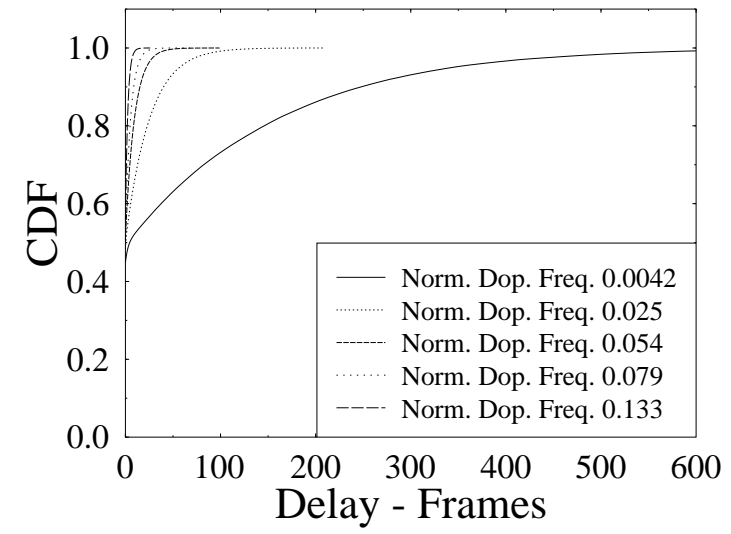

Figure 3: $\mathrm{CDF}$ of delay expressed in terms of $4.165 \mathrm{~ms}$ frames, for speech transmission with $\Xi$ of 0.6 and various normalised Doppler frequencies.

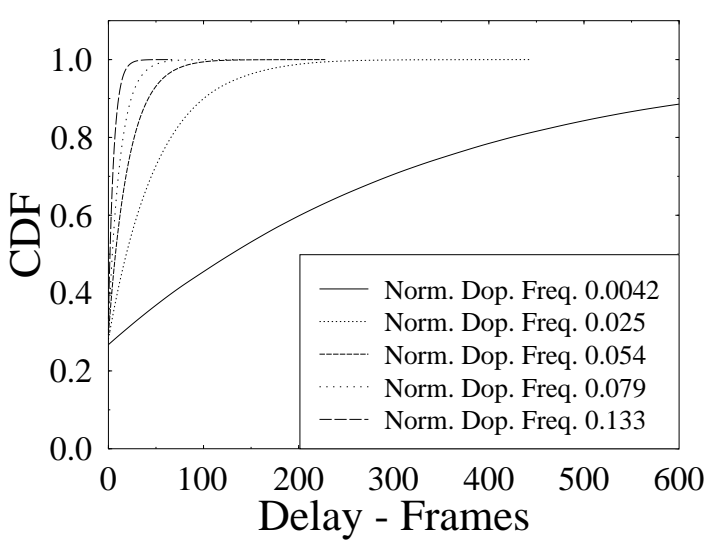

Figure 4: $\mathrm{CDF}$ of delay expressed in terms of $4.165 \mathrm{~ms}$ frames for computer data transmission with $\Xi$ of 0.6 and various normalised Doppler frequencies.

estimation and transmission. This is sufficiently low to support the assumption that the channel does not vary between estimation and transmission.

Accordingly, every $4.615 \mathrm{~ms}$ the TS to be employed by a particular user will be evaluated. The Doppler frequency, normalised to the transmission frame rate, will determine how often actually the employed TS will vary. Markov techniques were used to model the TS that would be employed. Transition probabilities between 'adjacent' TS schemes, such as BPSK and 4QAM may be evaluated analytically [8]. However, simulations showed that the probability of nonadjacent transitions, such as a BPSK frame followed by a 64QAM frame were not negligible for the system parameters of interest. Therefore, the transition probabilities were evaluated by simulation for average channel SNRs of 10, 20, 30,40 and $50 \mathrm{~dB}$, for $\Xi$ values of $0.6,0.7$ and 0.8 as well as for normalised Doppler frequencies of $0.0042,0.025,0.054$, 0.079 and 0.133 , using both speech and computer data sys- 
tem switching levels. At $2 \mathrm{GHz}$ and at a frame duration of $4.615 \mathrm{~ms}$ these normalised Doppler frequencies correspond to mobile velocities of $0.136,0.812,1.75,2.57$ and $4.32 \mathrm{~ms}^{-1}$.

\section{RESULTS}

Having obtained the above transition probabilities for the Markov models, experiments were conducted in order to generate the Cumulative Distribution Functions (CDFs) for the number of frames for which each bit was delayed. Figures 3 and 4 are examples of such delay CDFs, expressed in terms of the number of $4.615 \mathrm{~ms}$ frames for $\Xi=0.6$, for various normalised Doppler frequencies and for speech and data schemes, respectively.

Observe in the Figures that in both cases the delay increases with slower normalised Doppler frequencies. As expected, due to its more stringent BER, the computer data system also results in longer delays than the speech system. The effect of a lower normalised Doppler frequency is to prevent the TS changing very frequently and, therefore, there are relatively long periods where the transmission throughput is higher than the input rate, followed by relatively long periods of the reverse situation. This is what results in bursts of latency. At higher normalised Doppler frequencies the TS changes more frequently and the latency is reduced. We also examined the probability of the buffer becoming empty, which would result in a waste of capacity when the channel conditions are favourable. We found that in all simulations the total throughput was only marginally less than the target value of $\Xi \cdot B$.

For the speech system a maximum of four frames latency introduced by the modem was considered acceptable, which corresponded to less than $20 \mathrm{~ms}$, or one typical predictive analysis frame duration. The delay CDFs were processed in order to determine the proportion of bits that were transmitted within the acceptable four frame latency. For the computer data system no maximum delay was imposed, instead, the mean delay was considered. Figures 5, 6, 7 and 8 illustrate the these measures of latency for both systems as a function of normalised Doppler frequency and average channel SNR.

Figure 5 shows that an increased normalised Doppler frequency reduces the latency, particularly when the average channel SNR is low. This improvement results from the reduced number of adjacent 'No Transmission' frames. At $30 \mathrm{~dB}$, the probability of 'No Transmission' is very low and, therefore, even a highly correlated channel results in only a few adjacent 'No Transmission' frames. By considering the transition probabilities for the Markov model this can be confirmed. In a slow-fading channel, exhibiting a normalised Doppler frequency of 0.004170 at $10 \mathrm{~dB}$ average channel SNR, the probability 'No Transmission' was 0.192 and the probability of remaining in 'No Transmission', given that is the current TS is 0.980 . At $30 \mathrm{~dB}$ these probabilities became 0.0021 and 0.775 , respectively, which reflects the dramatic reduction in delay.

Observing Figure 6 reveals that the mean delay for the computer data is also reduced as the normalised Doppler frequency increases. It is interesting to note that for all $\Xi$ values and all average channel SNRs the shape of the curves in the Figure is similar. Bearing in mind the logarithmic nature of the $y$-axis, this Figure again shows that

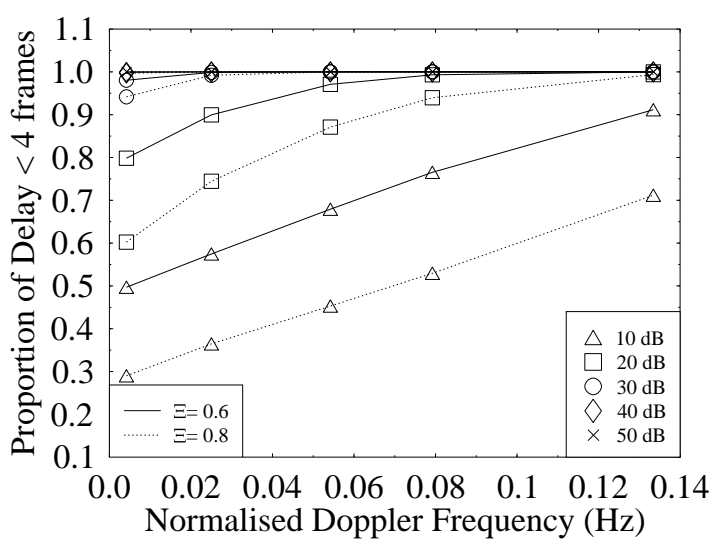

Figure 5: Proportion of transmitted bits in speech system, through a Rayleigh fading channel, experiencing four or fewer frames latency in the modem as a function of normalised Doppler frequency for, $\Xi$ of 0.6 and 0.8 , and average channels SNR of 10,20,30, 40 and $50 \mathrm{~dB}$

the increased de-correlation in the channel has greater effect upon the latency of systems under low average channel SNR conditions. Furthermore, most of the delay-reduction is observed due to the initial increases in normalised Doppler frequency.

Figure 7 shows how the performance of the fast and slow fading channels, associated with Doppler frequencies of 0.004170 and 0.133427 , respectively, converge at high average channel SNRs. This is because the instantaneous channel SNR is virtually always greater than $l_{4}$ and, therefore always $64 \mathrm{QAM}$ is employed, irrespective of the normalised Doppler frequency. At $20 \mathrm{~dB}$ average channel SNR the latency is almost independent of $\Xi$ for the faster fading channel, while for the lower fading rate there is nearly a factor of two performance difference between $\Xi$ values of 0.6 and 0.8 . This reflects the highly non-linear nature of the latency measurement.

Figure 8 show the convergence in latency performance for both fading rates at high average channel SNRs. The logarithmic nature of the $\mathrm{y}$-axis shows that an increased average channel SNR results in more significant benefits in latency for slower fading.

\subsection{Frequency Hopping}

The results above show that increased de-correlation in the fading channel reduces latency in an adaptive modulation scenario. Further de-correlation may be introduced by including frequency hopping into the system. This has no adverse effects upon the channel estimation when it is performed using passive reception, as outlined in the Introduction. The frequency hopping was simulated assuming $M$ independent fading channels, with a frequency hop every frame. All of the previous experiments were conducted with $M=2,3,5$ and 10 . Figures 9 and 10 show the effect of $M$ independent frequency hopped channels upon the latency for the speech and computer data transmission systems, for 10 


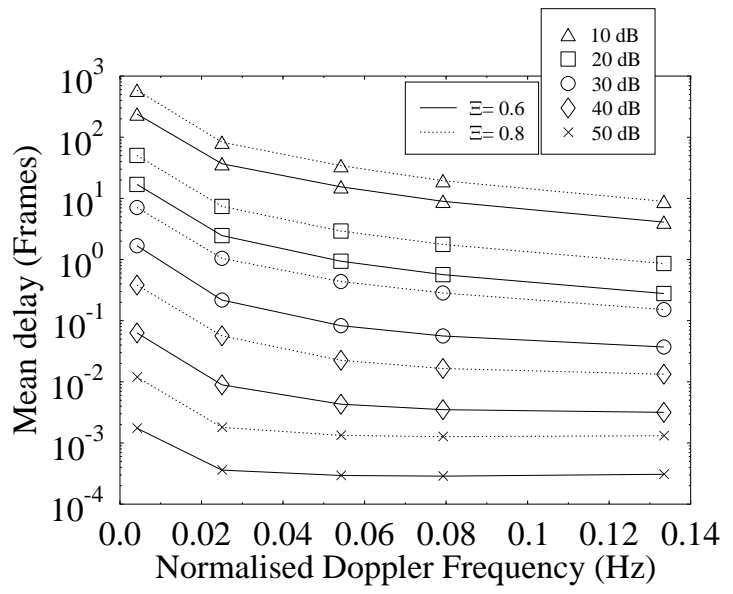

Figure 6: Mean delay of bits expressed in terms of the number of frames for the computer data transmission system, through a Rayleigh fading channel, as a function of normalised Doppler frequency, for $\Xi$ of 0.6 and 0.8 , and average channels SNR of $10,20,30,40$ and $50 \mathrm{~dB}$

and $20 \mathrm{~dB}$ average channels SNR, $\Xi$ values of 0.6 and 0.8 , and normalised Doppler frequencies of 0.004170 (Slow) and 0.133427 (Fast). These figures reveal that significant reductions in delay may be achieved by introducing frequency hopping to an adaptive modulation scheme. Ten hopping frequencies allow virtually all bits under all circumstances in the speech system to exhibit less than four frames redundancy. Observe in Figure 10 for the computer data system that all mean delays are reduced below 20 frames, which is equivalent to $92 \mathrm{~ms}$.

\section{CONCLUSIONS}

Previous work has shown that adaptive modulation can mitigate the effects of fading and maintain an arbitrary BER. This study has shown how the latency varies with $\Xi$, the average channel SNR and the normalised Doppler frequency. It was also demonstrated that frequency hopping reduces the latency, when the fading is very slow. These features provide further justification for the inclusion of adaptive modulation within a multi-mode UMTS air interface toolbox.

\section{ACKNOWLEDGMENT}

The financial support of the EPSRC, UK in the framework of the contract GR/K74043 is gratefully acknowledged.

\section{REFERENCES}

[1] R. Steele and W.T. Webb, "Variable rate QAM for data transmission over Rayleigh fading channels," in Wireless '91, Calgary, Alberta, pp. 1-14, IEEE, 1991.

[2] J. M. Torrance and L. Hanzo, "Adaptive modulation in a slow Rayleigh fading channel," in $7^{\text {th }}$ Personal, Indoor and Mobile Radio Communications (PIMRC) Conference, pp. 497-501, IEEE, 1996.

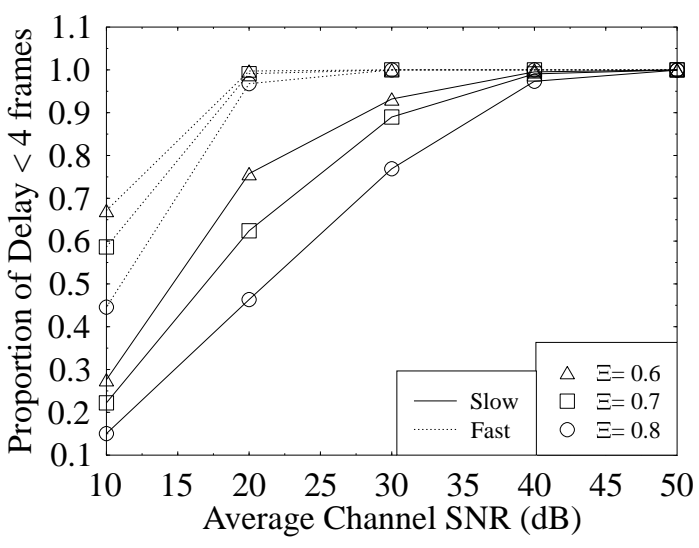

Figure 7: Proportion of transmitted bits in speech system, through a Rayleigh fading channel, experiencing four or fewer frames latency in the modem as a function of average channel SNR for $\Xi$ values of $0.6,0.7$ and 0.8 , and normalised Doppler frequencies of 0.004170 (Slow) and 0.133427 (Fast).

[3] J. M. Torrance and L. Hanzo, "Upper bound performance of adaptive modulation in a slow Rayleigh fading channel." Electronics Letters, April 1996. pp 169-171.

[4] Takashi Suzuki, Seiichi Sampei and Norihiko Morinaga, "Space and path diversity combining technique for $10 \mathrm{Mbits} / \mathrm{s}$ adaptive modulation/TDMA in wireless communications systems," in $46^{t h}$ Vehicular Technology Conference, pp. 1003-1007, IEEE, 1996.

[5] Soon-Ghee Chua and Andrea Goldsmith, "Variablerate variable-power mqam for fading channels," in $46^{\text {th }}$ Vehicular Technology Conference, pp. 815-819, IEEE, 1996.

[6] J. M. Torrance and L. Hanzo, "Optimisation of switching levels for adaptive modulation in a slow Rayleigh fading channel." Electronics Letters, June 1996. pp 1167 - 1169 .

[7] Francois de Ryck, Hakan Persson, Tom Leskinen, Tero Ojanpera and Werner Mohr, "FRAMES contribution to the standardisation of the umts air interface," in ACTS Mobile Telecommunications Summit, pp. 244249, IEEE, November 27-29, 1996.

[8] Hong Wang and Nader Moayeri, "Finite-state markov channel-a useful model for radio communication channels," IEEE Transactions on Vehicular Technology, vol. 44, no. 1, pp. 163-171, 1995. 


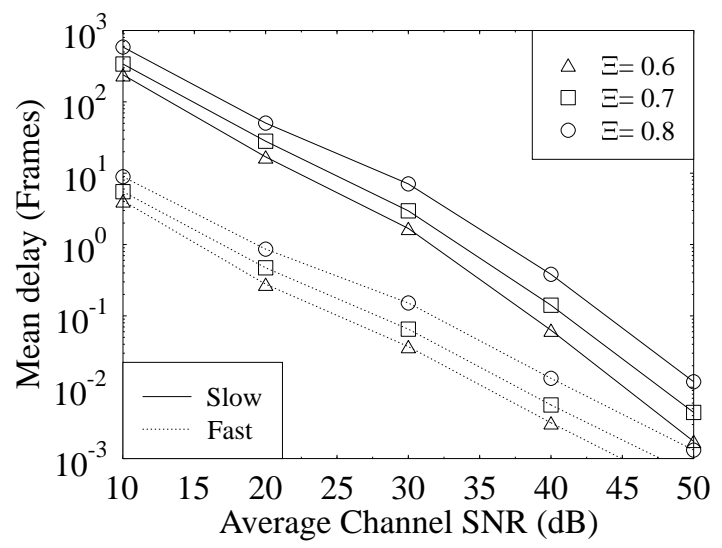

Figure 8: Mean modem delay expressed in terms of the number of frames for the computer data system, over a Rayleigh fading channel, as a function of average channel SNR for $\Xi$ values of $0.6,0.7$ and 0.8 , and normalised Doppler frequencies of 0.004170 (Slow) and 0.133427 (Fast).

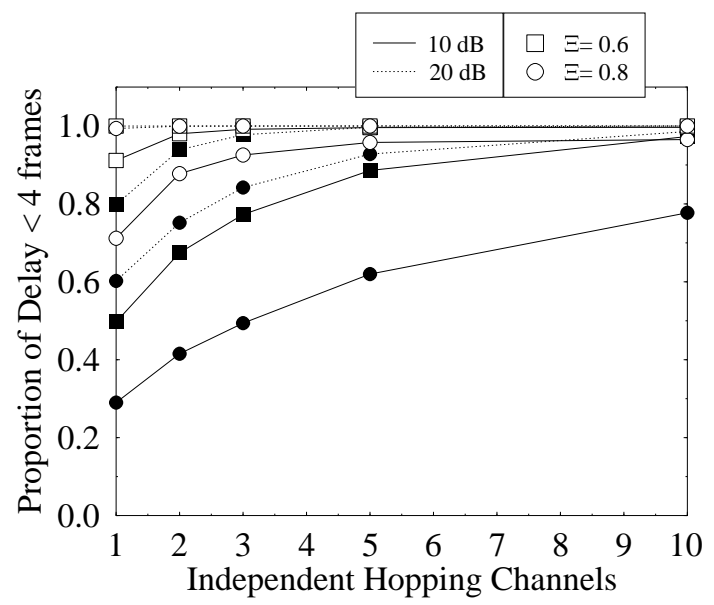

Figure 9: The effect independent frequency hopped channels upon the latency for the speech transmission system, for 10 and $20 \mathrm{~dB}$ average channels SNR, $\Xi$ values of 0.6 and 0.8 , and normalised Doppler frequencies of 0.004170 (Slow) and 0.133427 (Fast). Bold markers correspond to slow, hollow ones to fast fading.

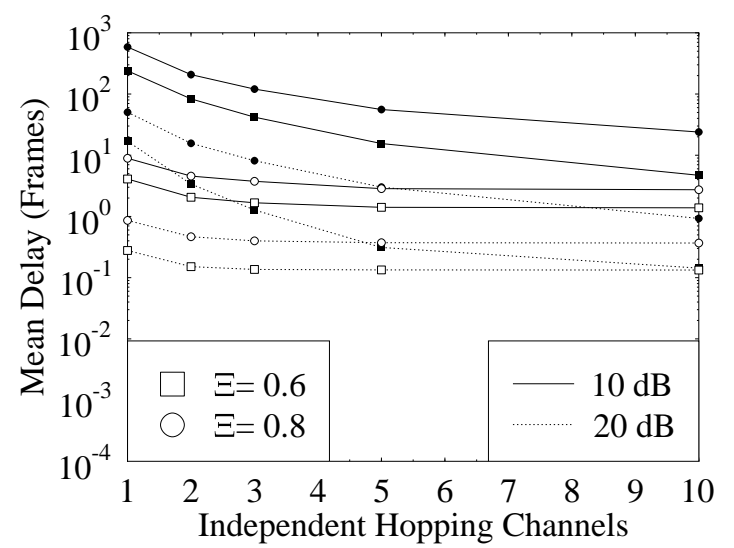

Figure 10: The effect independent frequency hopped channels upon the latency for the computer data transmission system, for 10 and $20 \mathrm{~dB}$ average channels SNR, $\Xi$ values of 0.6 and 0.8 , and normalised Doppler frequencies of 0.004170 (Slow) and 0.133427 (Fast). Bold markers correspond to slow, hollow ones to fast fading. 